PAPER

\title{
Influence of the frequency of sound pressure level on subjective loudness for a certain length of noise
}

\author{
Junji Yoshida* and Yuta Chaki \\ Osaka Institute of Technology, 5-16-1 Omiya, Asahi-ku, Osaka, 535-8585 Japan \\ (Received 16 February 2012, Accepted for publication 20 June 2012)
}

\begin{abstract}
In this study, we performed two subjective evaluation tests to obtain factors that affect subjective loudness for environmental noise having a certain duration. In the first experiment, a subjective loudness evaluation was performed using road- and rail-traffic noises having durations of $10 \mathrm{~min}$. As the result, rail-traffic noise was evaluated as being softer than road-traffic noise, although $L_{\text {Aeq }}$ of the rail-traffic noise was larger than that of road-traffic noise. Rail-traffic noise had longer duration of the low sound pressure level (SPL) and a short, higher SPL peak than road-traffic noise, and we concluded that the factor of the subjective loudness difference depended on the difference in the low SPL frequency. In the second experiment, subjective loudness evaluations were performed using road-traffic noise and modified road-traffic noise. In the modification, the SPL frequency of the road-traffic noise was changed to be similar to that of rail-traffic noise. As the result, the modified road-traffic noise was evaluated as being softer than the original road-traffic noise. In summary, the subjective loudness for a certain length of noise was found to be affected by the frequency of the SPL, and when noise has a long, low SPL duration, it is evaluated as being softer.
\end{abstract}

Keywords: A certain length of noise, Environmental noise, Road-traffic noise, Rail-traffic noise, Railway bonus, Memory, Frequency

PACS number: 43.50.Rq, 43.66.Cb [doi:10.1250/ast.34.19]

\section{INTRODUCTION}

The reduction of environmental noise is important for living comfortably. For the reduction of environmental noise, it is necessary to set and follow an appropriate environmental noise evaluation index in addition to reducing the sound source level from vehicles and installing sound insulation barriers. The equivalent continuous Aweighted sound pressure level $\left(L_{\mathrm{Aeq}}\right)$ is employed as the standard environmental noise evaluation index at present. This index is useful because it can be obtained easily. However, some studies have reported that this index does not express the sensation of human beings accurately in relation to the frequency characteristic of environmental noise, but no other suitable evaluation indexes for environmental noise evaluation have been developed until now [1-4]. In considering a suitable index for environmental noise, in addition to considering the influence of the frequency characteristic, the influence of evaluation duration on subjective loudness should be investigated because environmental noise is typically evaluated for a certain length of time.

*e-mail: yoshida@med.oit.ac.jp
Many studies have been performed on the subjective loudness of a certain length of noise [5-10]. For example, Hellbruck performed a subjective loudness evaluation test using environmental noise for $20 \mathrm{~min}$ (a certain length of noise) and separated the noise for $30 \mathrm{~s}$ (short-term noise). As a result, the subjective loudness of a certain length of noise was evaluated to be louder than the average subjective loudness of short-term noise [7]. Similar tendencies were observed in other studies [5,6]. These studies revealed the tendency of the effects of memory: a loud sound is remembered through hearing a certain length of noise, but a soft sound is lost in the memory. In contrast, it was reported that not only loud sounds could be memorized but also other sounds could be remembered depending on the sound content [9]. Hence, factors other than the loudness of the presented sound may have an influence on the overall subjective loudness for a certain length of noise.

Fastl et al. investigated the subjective loudness of road- and rail-traffic noise and found that rail-traffic noise was evaluated as being softer than road-traffic noise in a laboratory study, even though both type of noise had the same $L_{\text {Aeq }}$ (Railway bonus) [11-17]. They posited that the 
frequency characteristic difference in these two types of environmental noises accounted for the Railway bonus $[14,15]$. However, other acoustical factors of the Railway bonus have not yet been considered.

In this study, to investigate the subjective loudness for a certain length of noise, we performed two subjective loudness evaluation tests using road- and rail-traffic noise and modified road-traffic noise.

\section{EXPERIMENT 1}

In the first experiment, the participants listened to road-traffic and rail-traffic noise having durations of $10 \mathrm{~min}$ through binaural headphones and evaluated their loudness.

\subsection{Stimuli, Procedure and Participants}

In this test, we performed a subjective loudness evaluation test to investigate subjective loudness for a certain length of road- and rail-traffic noise. Here, we considered if only one road-traffic noise and one rail-traffic noise are used for the evaluation, sound factors other than the evaluation target (road- and rail-traffic noise), such as bird twittering, recorded for the measurement duration may affect the subjective loudness. Accordingly, we used four samples of road-traffic noise (road-traffic noise group) and four samples of rail-traffic noises (rail-traffic noise group) measured at various areas and times for the investigation and analyzed them all together in each group to reduce the influence of other factors. The four samples of road-traffic noise were measured for $10 \mathrm{~min}$ at a height of $150 \mathrm{~cm}$ at the side of a road, and the four samples of rail-traffic noise were measured for the same duration and at the same height near a railway. The presented noise (road- and rail-traffic noise) samples were measured using binaural headset microphones (HEAD Acoustics: SQuadriga). The values of $L_{\mathrm{Aeq}}$ for $10 \mathrm{~min}$ of measured road- and rail-traffic noise and the calculated loudness values according to DIN 45631/A1 are shown in Table 1.

As shown in Table $1, L_{\text {Aeq }}$ and the calculated loudness value of the measured environmental noises varied. Therefore, if we performed a subjective loudness evaluation test using these noise samples, the subjective loudness in each road- and rail-traffic noise group would be affected by the difference in $L_{\mathrm{Aeq}}$ or the calculated loudness. Also, rail-traffic noise was reported to be evaluated as being softer than road-traffic noise, even when both types of noise had the same $L_{\mathrm{Aeq}}$, depending on the frequency characteristics of loudness (Railway bonus) [14,15]. The calculated loudness is another index that can express subjective loudness considering the influence of the frequency characteristics. We compensated the amplitude of the measured road-traffic noise to match the calculated loudness. Therefore, the loudness was similar to that of railtraffic noise (13.5 \pm 1 sone). We performed the subjective
Table $1 L_{\text {Aeq }}$ and loudness values of each measured environmental noise.

\begin{tabular}{cccccc}
\hline Sound & $\begin{array}{c}L_{\text {Aeq }} \\
(\mathrm{dB})\end{array}$ & $\begin{array}{c}\text { Loudness } \\
(\text { sone })\end{array}$ & Sound & $\begin{array}{c}L_{\text {Aeq }} \\
(\mathrm{dB})\end{array}$ & $\begin{array}{c}\text { Loudness } \\
(\text { sone })\end{array}$ \\
\hline Road 1 & 73.3 & 25.9 & Rail 1 & 68.6 & 14.0 \\
Road 2 & 74.0 & 26.6 & Rail 2 & 69.3 & 12.7 \\
Road 3 & 74.3 & 27.5 & Rail 3 & 69.4 & 12.5 \\
Road 4 & 73.2 & 25.0 & Rail 4 & 70.2 & 12.5 \\
\hline
\end{tabular}

Table 2 Compensative $L_{\text {Aeq }}$ and loudness values of each measured environmental noise.

\begin{tabular}{cccccc}
\hline Sound & $\begin{array}{c}L_{\text {Aeq }} \\
(\mathrm{dB})\end{array}$ & $\begin{array}{c}\text { Loudness } \\
(\text { sone })\end{array}$ & Sound & $\begin{array}{c}L_{\text {Aeq }} \\
(\mathrm{dB})\end{array}$ & $\begin{array}{c}\text { Loudness } \\
(\text { sone })\end{array}$ \\
\hline Road 1 & 63.7 & 13.9 & Rail 1 & 68.6 & 14.0 \\
Road 2 & 64.0 & 13.8 & Rail 2 & 69.3 & 12.7 \\
Road 3 & 63.3 & 13.4 & Rail 3 & 69.4 & 12.5 \\
Road 4 & 64.1 & 13.8 & Rail 4 & 70.2 & 12.5 \\
\hline
\end{tabular}

loudness evaluation test using the compensated noise to investigate factors, other than the frequency characteristic, that influence the subjective loudness for a certain length of noise. The calculated values of $L_{\mathrm{Aeq}}$ and the loudness values after compensation are shown in Table 2.

In the experimental procedure, the environmental noises were saved in a personal computer (PC). One noise sample was selected randomly and presented to the experimental participant via headphones (Sennheiser: HD600) through a playback system (HEAD acoustics: PEQ 4). After the presentation of a certain length of noise (10 $\mathrm{min})$, the participant selected the subjective loudness from 31 levels from 0 (very soft) to 30 (very loud). In addition, short-term loudness evaluations were performed in $30 \mathrm{~s}$ intervals for the presentation duration of a certain length of noise $(10 \mathrm{~min})$ to check the participant's consciousness of the presented sound. For the short-term evaluation, a timer on the PC monitor was used to inform the participant of the evaluation timing. In other words, timing was conveyed without presenting any additional sound. The short-term evaluation for $30 \mathrm{~s}$ was performed a total of 20 times for each evaluation of a certain length of noise for $10 \mathrm{~min}$. The subjective loudness was evaluated in the same way as the evaluation of a certain length of noise. Also, the loudness evaluation test was performed separately for road- and rail-traffic noise in the first experiment.

Seven males in their 20's having normal hearing ability participated in the tests. Each participant evaluated loudnesses of 20 short-term noises at $30 \mathrm{~s}$ intervals and evaluated the loudness of a certain length of noise for the duration of $10 \mathrm{~min}$ (21 evaluations were performed for one test). The eight samples of environmental noise (four roadand four rail-traffic noise samples) were evaluated. There- 


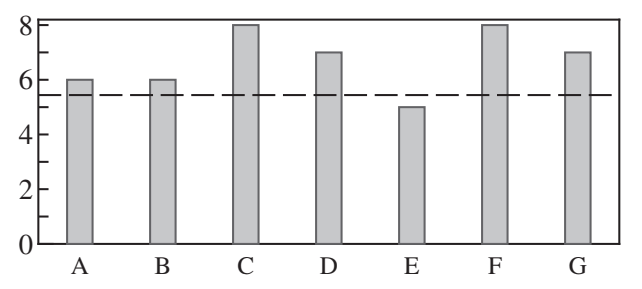

Fig. 1 Number of tests yielding a correlation coefficient above 0.75 between subjective loudness and $L_{\text {Aeq }}$ for short-term noise. The dashed line indicates the twothirds line of all tests.

fore, each participant performed eight tests, and a total of 56 tests were performed by the participants. Hence, 1,176 evaluations ( 21 evaluations $\times 56$ tests) were performed in total.

\subsection{Result}

Before analyzing the subjective loudness evaluation results for a certain length of noise, we checked the correlation between the subjective loudness and the $L_{\text {Aeq }}$ for short-term noise for each participant in each test to check the participants' evaluation ability for a certain length of noise. If the correlation coefficient was under 0.75 for at least one-third of all tests, the participant was considered not to be conscious of the presented sound for the experimental duration. Therefore, that participant's evaluation results were not used in the following analysis because the reliability of the evaluations for a certain length of noise was predicted to be low. Figure 1 shows the number of tests having a correlation coefficient of over 0.75 for each participant. The dashed line in the figure indicates the two-thirds line of all tests; therefore, the evaluation results of any participant falling below that line were excluded.

The result of participant $\mathrm{E}$ fell below the line, and the evaluation results for a certain length of noise of this participant were excluded. Therefore, the experimental results for a certain length of noise of six participants were used in the following analysis. Also, the correlation coefficient between the subjective loudness of the six participants and $L_{\text {Aeq }}$ was 0.92 .

Subsequently, the tendency of subjective loudness for a certain length of road- and rail-traffic noise was analyzed. The subjective loudness was analyzed all together in each road- and rail-traffic noise group to reduce the influence of the other sound factors included in the measurement for a certain length of road- and rail-traffic noise.

Figure 2 shows the average subjective loudness of the six participants for the certain length of noise group of road- and rail-traffic and the standard deviation.

As shown in Fig. 2, the subjective loudness of a certain length of rail-traffic noise was evaluated as being softer

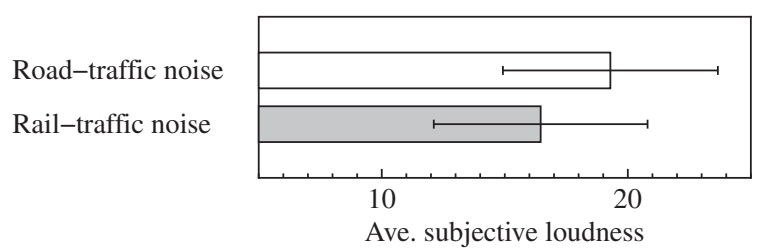

Fig. 2 Average subjective loudness of road- and railtraffic noise groups and the standard deviation.

than that of road-traffic noise, even though these types of noise had almost the same calculated loudness value, and $L_{\text {Aeq }}$ of rail-traffic noise was almost $5 \mathrm{~dB}$ higher than that of road-traffic noise. Next, the $t$-test was performed by regarding ordinal scales of the 31 levels as interval scales [18] to verify the difference in the subjective loudness for a certain length of noise between road and rail traffic. As a result, the loudness difference between road- and rail-traffic noise was significant when the significance level was set to be below 0.05 ( $p=0.029$ ).

This result is consistent with the experimental results obtained by Fastl et al., in which the loudness of rail-traffic noise was evaluated as being softer than that of road-traffic noise, even if the noise samples had similar $L_{\text {Aeq }}$ (Railway bonus) [11-17]. Fastl et al. considered that the Railway bonus occurred owing to the influence of the frequency characteristic on loudness as an acoustical factor [14,15]. The amplitudes of road- and rail-traffic noise samples used in this experiment were compensated to equalize their loudness values in consideration of the influence of the frequency characteristic, but the subjective loudness of rail-traffic noise for a certain length of noise was evaluated as being softer. This result indicates that the Railway bonus depends not only on the difference in the frequency characteristic, but also on another acoustical factor affecting the subjective loudness for a certain length of noise may exist.

Then, we focused on the time variation of each presented noise sample. Figure 3 shows the time variation of the SPL in road-traffic noise ( Road 1) and rail-traffic noise (Rail 1).

As shown in Fig. 3, rail-traffic noise (gray line) has a higher SPL than road-traffic noise, but the frequency is low, even though the average calculated loudness values were almost the same. Also, the frequency of the low SPL in rail-traffic noise was much higher than that in roadtraffic noise. Road-traffic noise had a high frequency at a mid-level SPL. This means the variance of the SPL distributions of rail-traffic noise is wider than that of road-traffic noise. This tendency was observed in other road- and rail-traffic noise samples. The probability of the SPL distribution and the standard deviation for each environmental noise are shown in Fig. 4. 


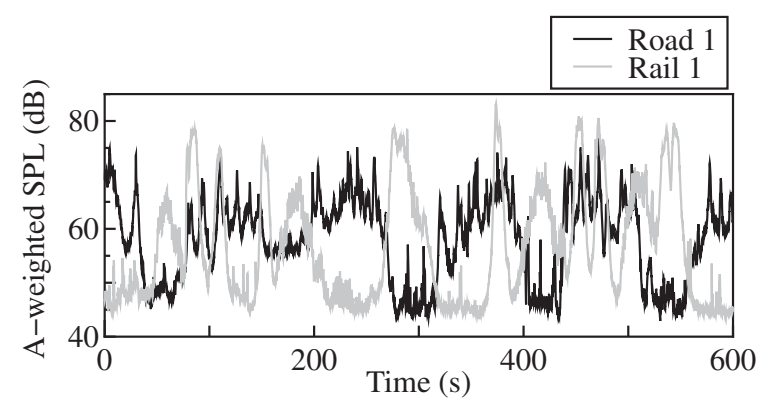

Fig. 3 Variation of sound pressure level of road- and rail-traffic noise in presentation duration.
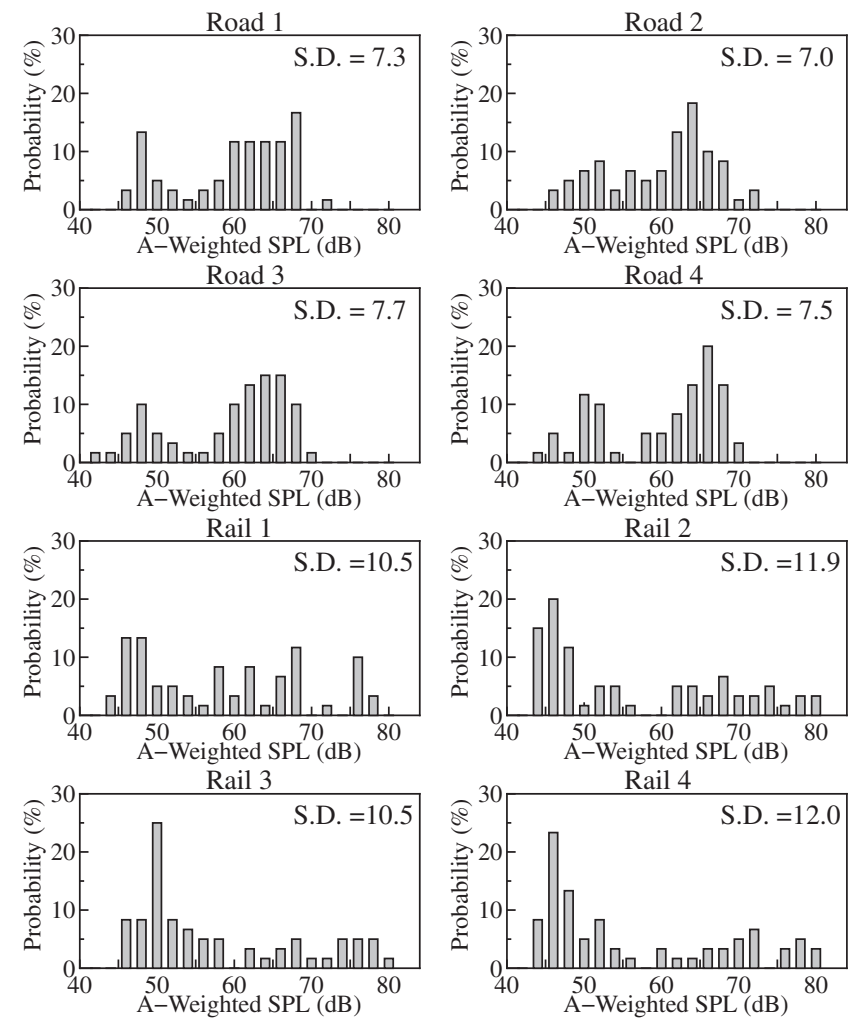

Fig. 4 Probability of sound pressure level and standard deviation of each road- and rail-traffic noise sample.

From these SPL distributions of road- and rail-traffic noise, the variations among all samples of rail-traffic noise were much wider than those in the case of road-traffic noise, and this tendency may decrease the subjective loudness for a certain length of noise.

\section{EXPERIMENT 2}

The subjective loudness of a certain length of railtraffic noise was evaluated as being softer than that of roadtraffic noise in Experiment 1. The high frequency of the low SPL in the presentation duration of rail-traffic noise (i.e., the wide SPL distribution) was considered to be one of the factors decreasing the subjective loudness. In this experiment, to verify the influence of the low SPL frequency on the subjective loudness, we modified the

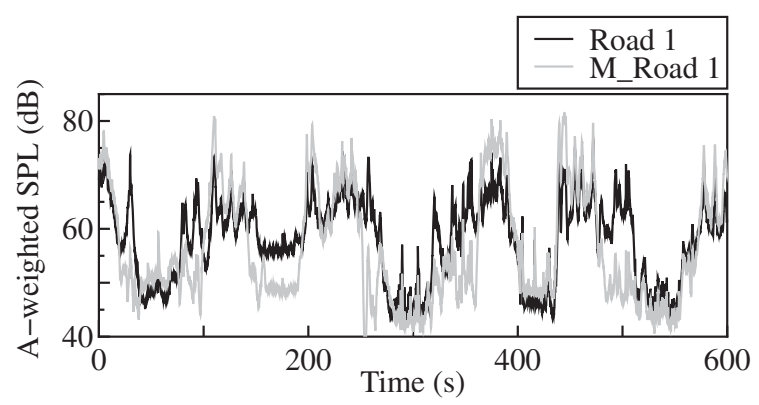

Fig. 5 Time variation of the SPL of original and modified road-traffic noise.

amplitude of road-traffic noise employed in Experiment 1 so that the SPL time variation was similar to that of rail-traffic noise, and then we performed the subjective loudness evaluation test using the original and modified road-traffic noises.

\subsection{Stimuli, Procedure and Participants}

The presented environmental noise samples were the four road-traffic noise samples used in Experiment 1 (original sound: Road 1 to Road 4) and four modified road-traffic noise samples (modified sound: M_Road 1 to M_Road 4) in which the time variations were modified to be almost the same as those of rail-traffic noise used in Experiment 1. Therefore, eight noise samples having 10 min durations were used for the subjective loudness evaluation. In the modification, the amplitude of the original road-traffic noise was changed in accordance with the time variation of rail-traffic noise. Rail-traffic noise used in Experiment 1 had a higher SPL peak and higher frequency of low SPL. Thus, the amplitude of the sound pressure of road-traffic noise was changed each time to be similar to that of rail-traffic noise. The amplitudes of roadtraffic noise samples 1, 2, 3, and 4 were modified referring to rail-traffic noise samples $1,2,3$, and 4, respectively. The time variation of the original road-traffic noise (Road 1$)$ and that of the modified road-traffic noise (M_Road 1) are shown in Fig. 5.

In addition, the loudness values calculated on the basis of DIN 45631/A1 were almost the same between the original and modified road-traffic noise upon changing the overall amplitude of the modified noise samples for analyzing the road- and modified road-traffic noise all together in each group (original road-traffic and modified road-traffic groups), the same as in Experiment 1. The distribution of the SPL and the standard deviation for each modified noise sample are shown in Fig. 6. After the modification, the standard deviation was increased about $3 \mathrm{~dB}$ in each road-traffic noise sample.

The value of $L_{\mathrm{Aeq}}$ for $10 \mathrm{~min}$ and the averaged loudness values for $10 \mathrm{~min}$ of the original and modified road-traffic noise samples are shown in Table 3. 

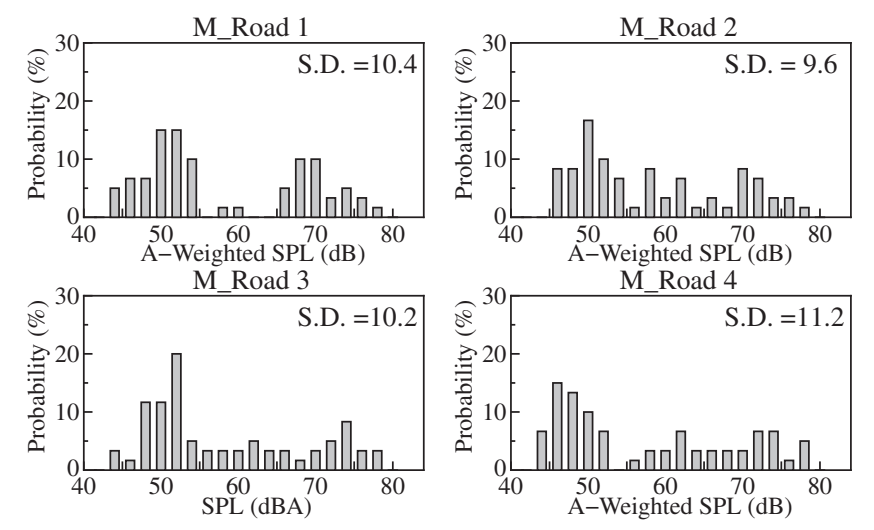

Fig. 6 Frequency sound pressure level and the standard deviation of modified road-traffic noise.

Table $3 L_{\text {Aeq }}$ and calculated loudness values of original and modified road-traffic noise samples.

\begin{tabular}{lccccc}
\hline Sound & $\begin{array}{c}L_{\text {Aeq }} \\
(\mathrm{dB})\end{array}$ & $\begin{array}{c}\text { Loudness } \\
(\text { sone })\end{array}$ & Sound & $\begin{array}{c}L_{\text {Aeq }} \\
(\mathrm{dB})\end{array}$ & $\begin{array}{c}\text { Loudness } \\
(\text { sone })\end{array}$ \\
\hline Road 1 & 63.7 & 13.9 & M_Road 1 & 67.6 & 13.7 \\
Road 2 & 64.0 & 13.8 & M_Road 2 & 67.3 & 13.7 \\
Road 3 & 63.3 & 13.4 & M Road 3 & 68.4 & 13.7 \\
Road 4 & 64.1 & 13.8 & M Road 4 & 68.7 & 13.2 \\
\hline
\end{tabular}

As the experimental procedure, one of the eight samples of environmental noise saved in the $\mathrm{PC}$ was selected randomly and presented to the participant via headphones (Sennheiser: HD600) through the playback system (HEAD acoustics: PEQ 4). After the presentation, the participant selected the subjective loudness from among the 31 levels from 0 (very soft) to 30 (very loud). In addition, a short-term loudness evaluation was performed as in Experiment 1 for each $30 \mathrm{~s}$ interval of the presentation duration of a certain length of noise $(10 \mathrm{~min})$. The short-term evaluations of $30 \mathrm{~s}$ were performed a total of 20 times for each evaluation of a certain length of noise. The subjective loudness evaluation was performed in the same way as that for a certain length of noise.

Fourteen males in their 20's having normal hearing ability participated in the tests. Seven of them participated in Experiment 1. Each participant evaluated the loudness of 20 short-term noises in each $30 \mathrm{~s}$ duration and evaluated the loudness of a certain length of noise for $10 \mathrm{~min}$ in a test (21 evaluations were performed in one test). In this experiment, the difference in the subjective loudness between the original and modified noise may be small because the original and modified noise samples were identical except for the time variation. Therefore, we iterated each test three times to obtain the significance difference in subjective loudness between the original and modified noise samples. Hence, 24 tests were per-

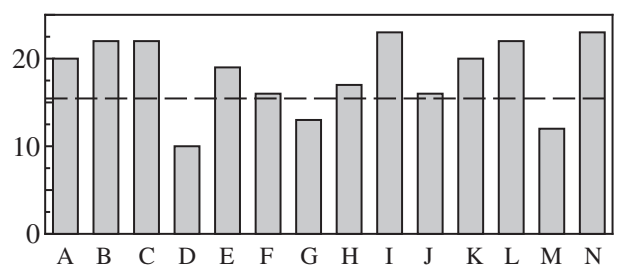

Fig. 7 Number of tests yielding a correlation coefficient above 0.75 between subjective loudness and $L_{\text {Aeq }}$ for short-term noise. The dashed line indicates the twothirds line of all tests.

formed for all eight presentation sounds for one participant, and a total of 336 tests (24 tests $\times 14$ participants) were performed for all participants. As a result, 7,056 evaluations $(21$ evaluations $\times 336$ tests $)$ were performed in total.

\subsection{Results}

Before analyzing the subjective loudness evaluation results for a certain length of noise, we calculated the correlation between the subjective loudness and $L_{\text {Aeq }}$ of short-term noise for each participant, as we did in Experiment 1 . Hence, a participant having a correlation coefficient below 0.75 in at least one-third of all tests was regarded as a participant not conscious of the presented sound for the experimental duration, and hence that participant's evaluation results were not used in the following analysis. Figure 7 shows the participants and the number of tests in which the correlation coefficient was above 0.75 . The dashed line in the figure indicates the two-thirds line of all tests; therefore, the evaluation results of any participant below the line were excluded.

As a result, three participants ( $D, G$ and $M$ ) were deemed not to be conscious of the presented sound in the tests, and so their evaluation results for a certain length of noise were excluded. Therefore, the experimental results for a certain length of noise of 11 participants were used in the following analysis. Also, the correlation coefficient between the subjective loudness for the 11 participants and $L_{\text {Aeq }}$ was 0.92 .

Figure 8 shows the subjective loudness averaged for the 11 participants in a group of a certain length of original and modified road-traffic noise and the standard deviation.

From the results, the subjective loudness of the modified noise was evaluated as being softer than that of the original sound. This means the loudness was evaluated as being softer after the modification in which the frequency of the low SPL was increased (i.e., the SPL distribution became wider). Then, a $t$-test was performed by regarding ordinal scales of the 31 levels as interval scales [18], to verify the subjective loudness difference between the original and the modified noises. As a result, the loudness difference 


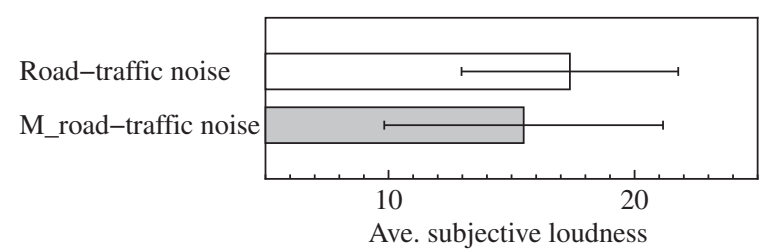

Fig. 8 Average subjective loudness of original and modified road-traffic noise groups and the standard deviation.

between the original and modified road-traffic noise samples was significant when the probability was set below $0.05(p=0.003)$. From the experimental results, the subjective loudness for a certain length of noise was found to be affected by the frequency of the SPL for the evaluated duration: when the frequency of the low SPL was high, the loudness was evaluated as being softer.

\section{SUMMARY AND DISCUSSION}

In this study, we performed a subjective loudness evaluation test using road- and rail-traffic noise in Experiment 1 to investigate the factors affecting the subjective loudness for environmental noise of a certain duration. As a result, rail-traffic noise was evaluated as being softer than road-traffic noise, even though these types of presented noise had similar loudness values according to DIN 45631/ A1. Therefore, we focused on the difference in the SPL distributions between rail- and road-traffic noise. Railtraffic noise had a higher maximum SPL but the frequency was low, and the low SPL had a much higher frequency in comparison with those of road-traffic noise.

In Experiment 2, we performed a subjective loudness evaluation test using the same road-traffic noise and modified road-traffic noise to verify the influence of the SPL distribution on subjective loudness. In the modification, the maximum SPL and the frequency of the low SPL were increased to be similar to those of the rail-traffic noise. As a result, the modified sound was evaluated as being significantly softer than the original sound.

From the above experimental results, the subjective loudness for a certain length of noise was found to be influenced by the SPL distribution for the presented duration, and when the frequency of the low SPL was high, the sound was evaluated as being softer.

The experimental results, such as environmental noise having a high frequency of a low SPL being evaluated as softer, may depend on the memory of the perceived sound, as described in previous studies [5-9]. In these other studies, the subjective loudness evaluation test was performed using noise of a certain length and shortterm noise, and the subjective loudnesses were compared. As a result, a certain length of noise was evaluated as being softer than short-term noise. The reason was presumed to be that loud sounds are remembered but soft sounds may be lost in hearing noise of a certain length.

In our study, the subjective loudness of rail-traffic noise for a certain length was evaluated as being softer than that of road-traffic noise, even though these sounds had almost the same calculated loudness values. The reason for the disagreement between the calculated loudness value and the subjective loudness for a certain length of noise is considered as follows. In the loudness evaluation for roadtraffic noise, the impression of soft sounds may be lost in the participant's memory through hearing the noise for a certain duration. On the other hand, when the participant evaluated the loudness of rail-traffic noise, low SPL of long duration existed in the presentation. Therefore, the impression of soft sounds might be remembered. Hence, when the participants evaluated rail-traffic noise, the impression of soft sound affects the overall loudness impression in addition to the impression of loud sound. As a result, a certain length of noise of the rail traffic was evaluated as being softer than road-traffic noise, in which the impression of soft sounds in road-traffic noise was lost in memory. In Experiment 2, the modified noise, in which the frequency of low SPL was increased, was evaluated as being softer than the original road-traffic noise for the same reason as stated above: the impression of soft sounds was not lost in memory owing to the increase in the frequency.

In addition, even though the values of $L_{\mathrm{Aeq}}$ of railtraffic noise were almost $5 \mathrm{~dB}$ higher than those of roadtraffic noise, the subjective loudnesses of rail-traffic noise were evaluated as being softer than those of road-traffic noise in Experiment 1. This tendency is almost the same as that of the Railway bonus [11-17]. The railway bonus has been reported from social surveys of environmental noise, and the nonacoustical factor is considered to be one of its factors $[11,12,16]$. Fastl et al. found that the Railway bonus could be observed in a laboratory test, and they discussed the Railway bonus as being caused by the difference in the frequency characteristics of noise samples $[14,15]$. In our study, the influence of the frequency characteristic on loudness in presented noise samples was compensated by changing the amplitudes to be similar to the calculated loudness value among the presented noise samples. However, the subjective loudness of rail-traffic noise was evaluated as being softer than that of road-traffic noise. This suggests the existence of another acoustical factor of the Railway bonus. As a possible factor, the time variation of the SPL was found to affect the subjective loudness for a certain length of noise, and when the frequency of the low SPL was high, the noise (rail-traffic noise) was evaluated to be softer than that (road-traffic noise) having a low frequency of the low SPL. However, the difference in the subjective loudness between road- and rail-traffic noise 
groups obtained in Experiment 1 was larger than that between the original and modified road-traffic noise groups in Experiment 2. This indicates that the characteristic of subjective loudness for a certain length of noise (Railway bonus) depends on not only the frequency of low SPL but also another factor such as a nonacoustical factor.

With respect to the frequency of low SPL, environmental noise of aircraft has high frequency of low SPL, the same as rail-traffic noise, but the community reaction to this noise in social surveys had been reported to be greater than to other types of traffic noise in contrast to the Railway bonus [11,16]. In another report, a survey about quietness in daily life revealed almost the same reaction to environmental noise, but a survey concerning annoyance indicated a much greater reaction than to other types of noise [19]. Also, the reaction to aircraft noise varied widely depending on community, even though $L_{\text {Aeq }}$ was almost the same [19]. From this tendency, it could be concluded that the difference of feeling between annoyance and loudness, and the nonacoustical factor of aircraft noise greatly contribute to increasing the reaction in spite of the noise having an acoustical characteristic evaluated as being softer because of having a very low SPL frequency. However, clarifying the factor behind the reaction to aircraft noise in detail is difficult at present and performing additional investigations is necessary in the future.

\section{REFERENCES}

[1] K. D. Kryter, "Scaling human reactions to the sound from aircraft," J. Acoust. Soc. Am., 31, 415-429 (1959).

[2] R. P. Hellman and E. Zwicker, "Why can a decrease in $\mathrm{dB}(\mathrm{A})$ produce an increase in loudness?," J. Acoust. Soc. Am., 82, 1700-1705 (1982).

[3] S. Namba, S. Kuwano, K. Kurakata and Y. Kikuchi, "Loudness and timbre of broad-band noise mixed with amplitudemodulated sounds," J. Acoust. Soc. Jpn. (E), 15, 131-142 (1994).

[4] J. Yoshida, "Consideration of new environmental noise evaluation index," Proc. ICA 2010, pp. 1-4 (2010).

[5] S. Namba and S. Kuwano, "The relation between overall noisiness and instantaneous judgment of noise and the effect of background noise level on noisiness," J. Acoust. Soc. Jpn. (E), 1, 99-106 (1980).

[6] H. Fastl, "Evaluation and measurement of perceived averaged loudness," Proc. 5th Oldenburg Symp. Psychological Acoustics, pp. 205-216 (1991).

[7] J. Hellbruck, "Memory of effects in loudness scaling of traffic noise - How overall loudness of short-term and long-term sounds depends on memory—," Acoust. Sci. \& Tech., 21, 329332 (2000).

[8] T. Kato, S. Kuwano and S. Namba, "A new approach to the memory of auditory events," Proc. 17th Int. Assoc. Empirical Aesthetics, pp. 349-354 (2002).

[9] S. Kuwano and S. Namba, "Memory of the loudness of sounds in relation to overall impression," Acoust. Sci. \& Tech., 24, 193-196 (2003).

[10] M. Morinaga, H. Tsukioka, J. Kaku, S. Kuwano and S. Namba, "The effect of off-time length on the overall impression of intermittent noise," Inter-noise 2011, pp. 1-8 (2011).

[11] J. M. Field and J. G. Walker, "Comparing the relationships between noise level and annoyance on different surveys: A railway noise vs. aircraft and road traffic comparison," $J$. Sound. Vib., 81, 51-80 (1982).

[12] U. Mohler, "Community response to railway noise: A review of social surveys," J. Sound Vib., 120, 321-332 (1988).

[13] H. Fastl, S. Kuwano and S. Namba, "Psychoacoustics and rail bonus," Inter-noise 1994, Vol. 2, pp. 821-826 (1994).

[14] H. Fastl, S. Kuwano and S. Namba, "Assessing in the railway bonus in laboratory studies," J. Acoust. Soc. Jpn. (E), 17, 139148 (1996).

[15] H. Fastl, "Masking effects and loudness evaluation," in Recent Trends in Hearing Research, H. Fastl, S. Kuwano and A. Schick, Eds. (Bibliotheks- und Informationssystem der Universität Oldenburg, Oldenburg, 1996), pp. 29-50.

[16] H. M. E. Miedema and H. Vos, "Exposure-response relationships for transportation noise," J. Acoust. Soc. Am., 104, 34323445 (1998).

[17] H. Fastl, M. Fruhmann and S. Ache, "Railway bonus for sounds without meaning?," Acoust. Aust., 31, 99-101 (2003).

[18] S. Namba and S. Kuwano, Method of Psychological Measurement for Hearing Research (Corona Publishing, Tokyo, 1998), pp. 72-86.

[19] M. Kadoya, T. Suda, M. Shouji and S. Sueoka, "Social response investigation for noise (No 5)," Annu. Rep. The Tokyo Metropolitan Research Institute for Environmental Protection 2007, pp. 97-104 (2007). 